\title{
Preparation and Properties of Copper Fine Wire on Polyimide Film in Air by Laser Irradiation and Mixed-Copper-Complex Solution Containing Glyoxylic Acid Copper Complex and Methylamine Copper Complex
}

\author{
Tomoji Ohishi*, Naoki Takahashi \\ Department of Applied Chemistry, Faculty of Engineering, Shibaura Institute of Technology, Tokyo, Japan \\ Email: *tooishi@sic.shibaura-it.ac.jp
}

How to cite this paper: Ohishi, T. and Takahashi, N. (2018) Preparation and Properties of Copper Fine Wire on Polyimide Film in Air by Laser Irradiation and Mixed-Copper-Complex Solution Containing Glyoxylic Acid Copper Complex and Methylamine Copper Complex. Materials Sciences and Applications, 9, 859-872.

https://doi.org/10.4236/msa.2018.911062

Received: August 9, 2018

Accepted: October 8, 2018

Published: October 11, 2018

Copyright $\odot 2018$ by authors and Scientific Research Publishing Inc. This work is licensed under the Creative Commons Attribution International License (CC BY 4.0).

http://creativecommons.org/licenses/by/4.0/

\begin{abstract}
Formation of copper wiring on a polyimide film by laser irradiation to a stable copper-complex film consisting of glyoxylic acid copper complex and methylamine copper complex in air has been investigated. A stable metallic copper on the polyimide film was precipitated even in air. Since this copper was generated only in the laser-irradiated parts, direct patterning of copper wiring was possible. It was also found that copper was precipitated by electroless copper plating on the laser-deposited copper wiring and it was possible to thicken the copper wiring by this precipitation. The resistivity of the copper wiring was almost the same as that of the bulk of metallic copper. The developed method-combining laser irradiation to a copper-complex-coated film and electroless copper plating-enables the high-speed deposition of fine copper wiring on a polyimide film in air by a printing process, indicating an inexpensive and useful process for fabricating copper wiring without high vacuum facility and heat-treatment under inert gas.
\end{abstract}

\section{Keywords}

Glyoxylic Acid Copper Complex, $\mathrm{CO}_{2}$ Laser, Fine Copper Wire, Laser Direct Patterning, Polyimide Film, Printable Electronics

\section{Introduction}

In recent years, a method called "printable electronics" - which produces electronic devices using a low-cost printing method-has been attracting much at- 
tention [1] [2] [3]. Compared with conventional sputtering, vacuum evaporation, CVD, and other methods using conventional high-vacuum equipment, printable electronics make it possible to form films by using simple equipment under atmospheric pressure. It is thus an attractive, innovative method for reducing the costs of manufacturing electronic devices. Even in regard to the formation of metal wiring, which is an indispensable component for electronic devices, the wiring is formed by a printing method using fine metal particles. Studies on the formation of gold and silver wiring have been reported; however, these wirings were formed by methods such as screen printing and inkjet printing using a highly dispersed solution of ultrafine particles of gold or silver [4]-[10]. And since gold and silver are precious metals, methods using them are problematic from the viewpoint of cost reduction. Although silver has high conductivity and is less expensive than gold, it has a problem in terms of its low migration resistance. For that reason, forming copper wiring by using a liquid dispersion of copper fine particles, which is a base metal with high conductivity, is being studied [11] [12]. However, copper is easily oxidized, so it is indispensable to perform the processing steps involved in this method-such as synthesis of copper fine particles, preparation and handling of their dispersion solution, and heat treatment necessary for film curing-under an inert gas [13] [14]. For that reason, the manufacturing process and its equipment are inevitably complicated, and that complexity is one reason for increasing costs. Therefore, the development of a stable and inexpensive copper-source compound, and a simple process technology for copper-wiring formation using it, is being eagerly anticipated.

Aiming to develop a simpler method for fabricating copper interconnects (wire), we are studying the formation of high-speed fine wire in air by using stable copper-metal complexes and laser irradiation. It has been reported that stable metallic copper is precipitated in air by laser irradiation of a copper-complex film having glyoxylic acid bonded as a ligand and that it is possible to perform direct drawing ("direct patterning") of copper wiring by utilizing the phenomenon by which copper precipitates only in the laser-irradiated part of the film [15] [16]. This method makes it possible to form fine copper wiring at high speed by laser irradiation of a copper metal complex that is stable and easy to handle in air; accordingly, it is attracting attention as a simple and low-cost technology for forming copper wiring that does not require high-vacuum equipment or treatment under inert gas.

Recently, formation of a copper film by using a stable solution composed of copper formate and 2-amino-2-methyl-1-propanol (AMP) as a copper source has been reported [17]; however, for conversion to copper, heat treatment at about $350^{\circ} \mathrm{C}$ under an inert gas is necessary. Such heat-treatment at high temperature is not suitable for forming copper wire on organic film. A method of forming a copper film with relatively good conductivity by irradiating a copper salt (as ink) with a high-intensity pulsed light has also been reported [18] [19]. 
However, direct patterning of copper wiring by photoirradiation on organic film in air has not been investigated and reported.

In this study, the formation of copper wiring on a polyimide film by our newly-developed method was studied. Polyimide film is a functional organic polymer with outstanding properties, namely, high heat resistance, electrical insulation, and low dielectric constant, and has been used as an organic film for printed circuit (wiring) boards [20] [21]. In particular, with the development of flexible electronics in recent years, demand for flexible printed circuit (wiring) boards has been growing. Conventionally, as for the mainstream method for fabricating a flexible printed wiring board, a copper foil is formed on a polyimide film, and copper wiring is formed from that foil by photolithography [22] [23] [24] [25] [26]. However, this method involves many steps, which complicate the manufacturing process and increase costs. Accordingly, a simpler method for forming copper wiring on a polyimide film is required. In consideration of the above-mentioned problems with the conventional method, we studied forming fine copper wiring on a polyimide film by the newly-developed method.

This paper reports the results of our investigation on direct patterning of fine copper wiring on a polyimide film by laser irradiation to the stable mixed-copper-complex film and the observation results (the surface state and cross-sectional structure of the formed copper film) concerning thickening of the copper wiring by electroless copper plating on the laser-deposited copper wiring.

\section{Experimental}

\subsection{Synthesis of Glyoxylic-Acid Copper (GACu) Complex}

Glyoxylic acid (4.5 mmol) was dissolved in $5 \mathrm{ml}$ of $\mathrm{H}_{2} \mathrm{O}$, and the solution was adjusted to $\mathrm{pH} 7$ by adding $10-\mathrm{wt} \% \mathrm{NaOH}$ aqueous solution. To that solution, $\mathrm{CuSO}_{4} \cdot 5 \mathrm{H}_{2} \mathrm{O}(4.5 \mathrm{mmol})$ dissolved in $5 \mathrm{ml}$ of $\mathrm{H}_{2} 0$ was added. After stirring that solution for three hours, the precipitated blue precipitate was filtered and washed with sufficient $\mathrm{H}_{2} \mathrm{O}$. The blue precipitate ( $\mathrm{GACu}$ complex) was then dried under reduced pressure. The yields in terms of weight and proportion were $0.50 \mathrm{~g}$ and $65.7 \%$, respectively.

\subsection{Synthesis of Methylamine-Copper (MACu) Complex}

To prepare a methylamine copper (MACu)-complex solution (1.0 M), $6.0 \mathrm{~mL}$ of $40 \%$ methylamine/methanol solution was added to $1.3 \mathrm{~g}$ of copper-formate tetrahydrate and stirred (with warming) for one hour with a stirrer. The MA$\mathrm{Cu}$-complex solution was gradually concentrated, and the solvent was removed and dried to obtain blue crystals.

\subsection{Preparation of Mixed Solution of Copper Complexes}

To prepare a GACu solution $(1.0 \mathrm{M}), 4.0 \mathrm{~mL}$ of ethanol and $2.0 \mathrm{~mL}$ of 2 -aminoethanol were sequentially added to $1.0 \mathrm{~g}$ of GACu and stirred with a stirrer for one hour. 
In addition, to prepare a methylamine copper (MACu) complex solution (1.0 $\mathrm{M}), 6.0 \mathrm{~mL}$ of a $40 \%$ methylamine/methanol solution was added to $1.3 \mathrm{~g}$ of copper-formate tetrahydrate and stirred with a stirrer for one hour. To prepare a mixed $(\mathrm{GACu} / \mathrm{MACu})$ solution $(1.0 \mathrm{M})$ of complexes, the (MACu) complex solution was added to the GACu complex solution and stirred for 30 minutes. To prepare a film-preparation-solution, mixing ratios (GACu:MACu) were varied as $10: 0,8: 2,6: 4,5: 5,4: 6,2: 8$, and $0: 10$.

\subsection{Thin-Film Preparation and Laser Irradiation}

The GACu/MACu mixed solution was spin coated (rotational speed: $2000 \mathrm{rpm}$; $30 \mathrm{~s}$ ) on a polyimide film (Kapton $100 \mathrm{H}$, Torey-DuPont, $20 \mathrm{~mm} \times 25 \mathrm{~mm} \times 25$ $\mu \mathrm{m}(\mathrm{t}))$ and sodium-free-glass substrate $(30 \mathrm{~mm} \times 30 \mathrm{~mm} \times 1 \mathrm{~mm}(\mathrm{t}))$, and the spin-coated films were then dried at $80^{\circ} \mathrm{C}$ for $10 \mathrm{~min}$. The polyimide film was surface-modified by irradiating the surface with ultraviolet rays. The thin film was irradiated by a carbon-dioxide-gas laser (wavelength $10.6 \mu \mathrm{m}$; SUNX LP-300) whose output was varied. The irradiation was performed with the laser focus swept at a sweep rate of $20 \mathrm{~mm} / \mathrm{s}$. After the laser irradiation, to remove non-irradiated portions, etching was performed with a mixed solvent of ethanol/ $\mathrm{H}_{2} \mathrm{O}$ at $1: 1$ or pure water, and then the thin film was washed with acetone and dried at room temperature.

\subsection{Electroless Copper Plating}

Copper deposition by electroless copper plating was performed on copper wiring fabricated by laser irradiation. The electroless-copper-plating solution was prepared by using EPITHAS PHP (pH 7.7) and Thru-Cup ELC-SP (pH 12), manufactured by Uemura Kogyo Co., Ltd., and the samples were formed by changing the immersion time of the thin films in the solution at a temperature of $60^{\circ} \mathrm{C}$.

\subsection{Evaluation}

The thermal-decomposition characteristics of the synthetic-complex were measured using thermogravimetric differential thermal analysis (TG-DTA; Shimadzu TGA-50) and thermogravimetric analysis mass spectrometry (TG-MS; RIGAKU TG 8120 and Shimadzu GCMS-QP 2010). The crystal structure of the thin film was measured with a thin-film X-ray diffractometer (RIGAKU RINT-TTR III, Smart Lab.).

The surface condition of the thin film was observed with a digital microscope (KEYENCE VHX-500F) and a scanning electron microscope (SEM; KEYENCE VE-8800 and JEOL SM-7610F). The cross-sectional structure of the copper thin film formed on the polyimide film was observed with a transmission electron microscope (TEM; Hitachi H-9500), the samples for which were formed by focused-ion-beam (FIB) processing. The resistivity of the thin film was measured by the four-end needle method (Mitsubishi MCP-T360). The adhesion strength of the thin film was evaluated by a JIS-standard tape test (JIS K5600-5-6: Japan 
Industrial Standard).

\section{Results and Discussion}

\subsection{Fabrication of Thin Film Using Glyoxylic-Acid Copper (GACu) Complex and Methylamine Copper (MACu) Complex and Effect of Laser Irradiation}

As a starting material for a copper source, the complex solution prepared by mixing $\mathrm{GACu}$ and $\mathrm{MACu}$, which are stable copper-divalent metal complexes, was used. The molecular structure of GACu and MACu is shown in Figure 1. A thin film was formed by changing the composition ratio of both complexes, and the state of the film after laser irradiation was observed.

The thin-film fabrication process is shown in Figure 2. A solution (with a concentration of $1 \mathrm{M}$ ) containing a mixture of a glyoxylic-acid copper complex (GACu) solution (solvent: 2-aminoethanol/ethanol mixed solvent) and methylamine copper complex ( $\mathrm{MACu}$ ) solution (solvent: methanol solution), and thin films formed with mixture ratios (GACu:MACu) of 10:0, 8:2, 6:4, 5:5, 4:6, 2:8, and 0:10 were examined. A copper-complex thin film was prepared by spin-coating (2000 rpm/30 s) a copper-complex solution on a glass slide or a polyimide film and pre-baking at $80^{\circ} \mathrm{C}$ for 10 minutes. The complex film was irradiated with $\mathrm{CO}_{2}$ laser in the atmosphere (laser power: $2.4 \mathrm{~W}$ at focal length; sweep rate: $20 \mathrm{~mm} / \mathrm{s}$ ) and etched to form copper wiring. The whole surface of the film was fabricated by moving the laser sweep laterally. At laser power of 1.2 $\mathrm{W}$, powder-like $\mathrm{Cu}$ particles with weak adhesion to polyimide film were deposited. At laser power of $2.4 \mathrm{~W}$ or more, $\mathrm{Cu}$ thin film with strong adhesion was formed. At laser power of $5.0 \mathrm{~W}$, polyimide film was deformed. To avoid

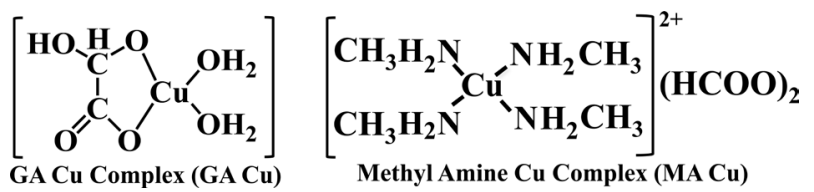

Figure 1. Molecular structures of glyoxylic acid copper complex (GACu) and methylamine copper complex (MACu).

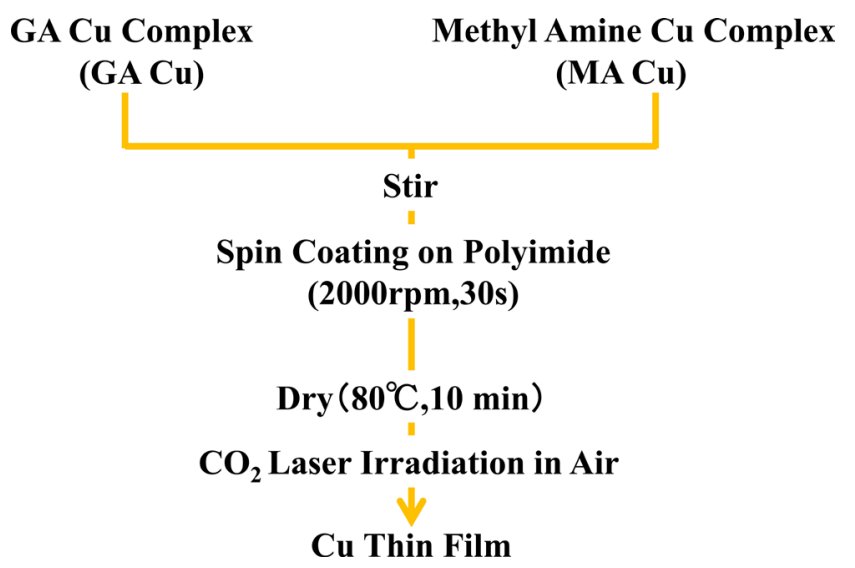

Figure 2. $\mathrm{Cu}$ film formation process using mixed solution of $\mathrm{GACu}$ and $\mathrm{MACu}$. 
damaging the polyimide film (which is an organic film) by the laser irradiation, the power of the laser irradiation was set low $(2.4 \mathrm{~W})$. In the thin films with any composition, the laser-irradiation site changed to copper color.

X-ray diffraction spectra obtained from the thin films are shown in Figure 3. Peaks appeared at $2 \theta=43.3^{\circ}(1,1,1), 50.4^{\circ}(2,0,0)$, and $74.2^{\circ}(2,2,0)$ in the case of all thin films irrespective of the compositional change. These peaks coincide with the cubic-copper peak. However, no peaks corresponding to $\mathrm{CuO}$ were observed. This result suggests that pure copper (i.e., no copper oxide) is precipitated in the laser-irradiated part of any of the copper-complex films with any composition.

The results of observation of the surface condition (surface morphology) of the copper wiring are shown in Figure 4. A copper wiring with a width of 200 $230 \mu \mathrm{m}$ was formed by laser sweep. As for the surface observation with a digital microscope (DM), the laser-sweep traces on a single GACu film were probed, and as the composition ratio of $\mathrm{MACu}$ increased, the sweep trace gradually disappeared, and in the case of the film with GACu:MACu $=5: 5$, a film with a uniform surface was obtained. As shown in the SEM images, many fine pores are present in the copper film prepared with the glyoxylic-acid-only copper complex (GACu:MACu = 10:0). When the methylamine copper complex was added, the size and number of the fine pores gradually decreasesd, and a relatively dense film was formed at GACu:MACu = 5:5. However, when the ratio of MA copper complex exceeded $50 \%$, surface irregularities and fine pores were increasingly

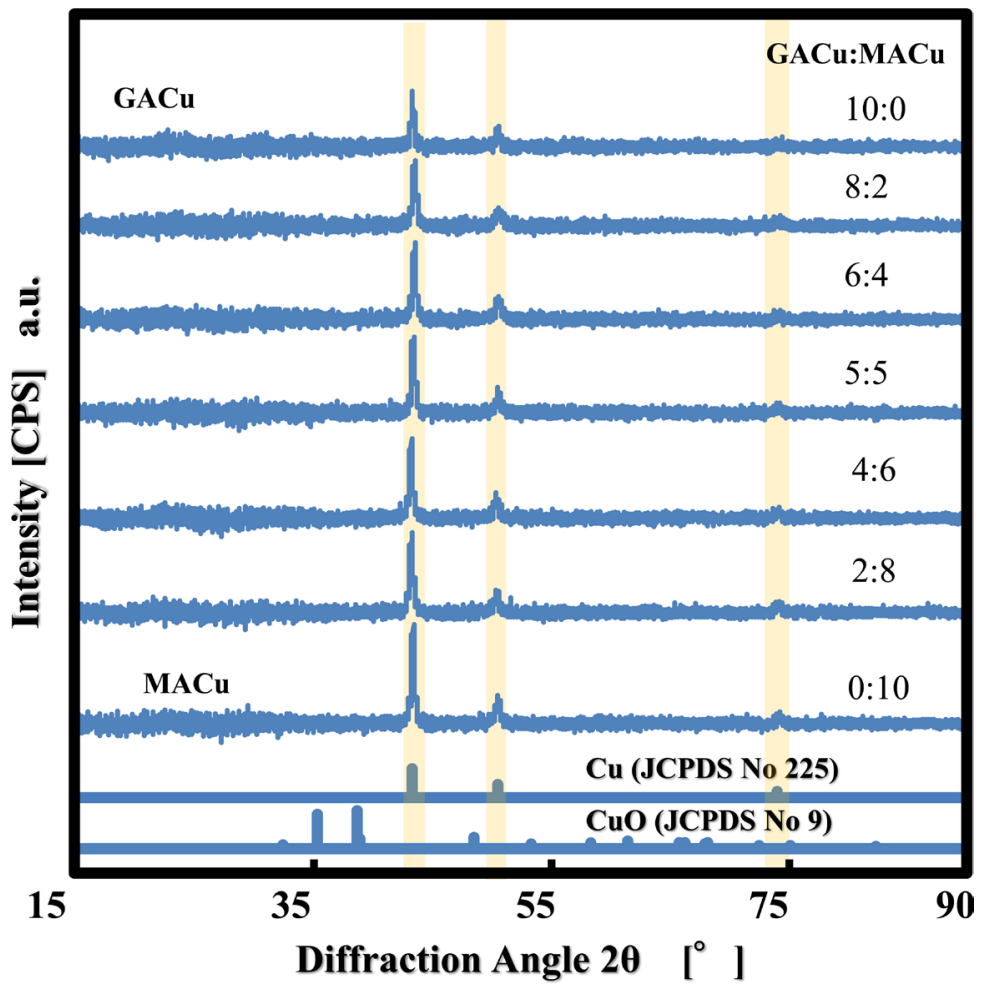

Figure 3. X-ray diffraction patterns of $\mathrm{Cu}$ thin films using mixed complex solution of GACu:MACu (Laser irradiation condition: laser power $2.4 \mathrm{~W}$, sweep speed $20 \mathrm{~mm} / \mathrm{s}$ ). 


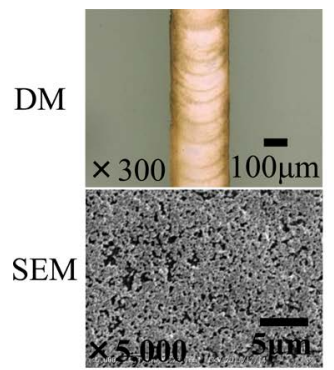

10:0

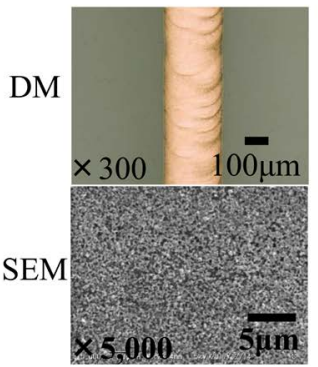

$4: 6$

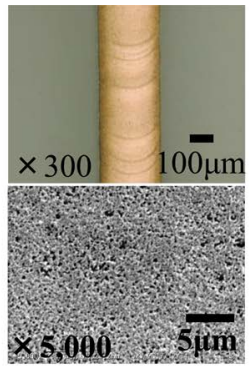

8:2

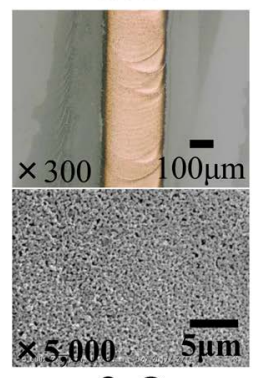

$2: 8$

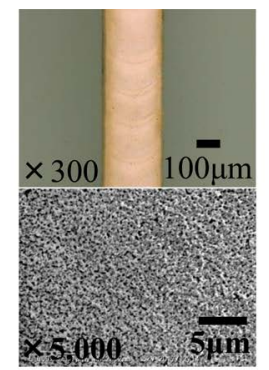

$6: 4$

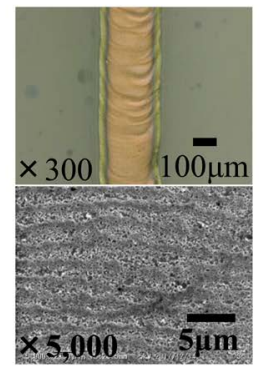

0:10

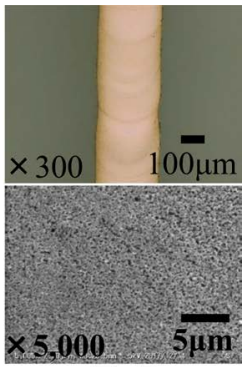

$5: 5$

Figure 4. Surface morphology of $\mathrm{Cu}$ fine wire using mixed complex solution of GA$\mathrm{Cu}: \mathrm{MACu}$ (Laser irradiation condition: laser power $2.4 \mathrm{~W}$, sweep speed $20 \mathrm{~mm} / \mathrm{s}$ ).

generated as the ratio of MA copper complex increased. In the case of the film containing only the MA copper complex, the surface roughness was greatest while the laser-sweep trace was largest. Moreover, when the MA-copper-complex ratio was increased (GACu:MACu $=2: 8$ and 0:10), the adhesion strength of the film to the substrate tended to decrease.

It was found that 1) copper metal is precipitated without oxidation of copper in air by laser irradiation to the two-component copper-complex film and 2) the surface state of the copper film largely depends on the mixing ratio of the two copper-complex components, and an optimal composition ratio for obtaining a dense film exists. Furthermore, by using a two-component system, copper is precipitated even at a low laser-power output of $2.4 \mathrm{~W}$, so it is possible to reduce thermal damage to the substrate caused by the laser. Accordingly, it is possible to form copper wiring on a film made of a resin such as polyimide. Although the mechanism of formation of a copper thin film by laser irradiation to the copper-complex film in air has already been reported [15], it is presumed to be explained as follows.

A $\mathrm{CO}_{2}$ laser with a wavelength of $10.6 \mu \mathrm{m}$ is an infrared laser, and it can apply heat with a high energy density locally and instantaneously to the irradiated part. The heat instantaneously detaches the ligands and decomposes the irradiated part. At that time, electrons are provided to $\mathrm{Cu}^{2+}$ ions by reductive elimination of the ligands, and those ions are reduced to copper. It is assumed that since the copper formed immediately after the reduction exists as very minute particles, the melting point of copper is extremely low compared with that of the bulk copper [27] [28]. It is considered that the fine copper particles melt due to the heat of the laser irradiation, and they grow into larger stable copper particles 
before they are oxidized. Mixing MACu with GACu when copper forms in this manner cause $\mathrm{MACu}$ to thermally decompose at a lower energy (According to an analysis of thermal-decomposition properties, ligand elimination of $\mathrm{MACu}$ is mostly completed at $200^{\circ} \mathrm{C}$, whereas that of $\mathrm{GACu}$ is complete at $270^{\circ} \mathrm{C}$ [16]). Consequently, when fine copper formed from $\mathrm{MACu}$ acts as a crystal nucleus, copper produced from GACu grows as crystals easily. This phenomenon is presumed to be the cause of the formation of a uniform copper film under a lower laser output in the case of the GACu/MACu-mixed-complex film rather than in the case of the GACu-only film.

On the other hand, in the case of heat treatment, thermal energy is gradually applied to the entire film by convection. It is therefore presumed that fine copper particles generated when the ligands are reductively eliminated react immediately with oxygen in the air diffused into the film without any grain growth occurring, and copper oxide is subsequently formed.

\subsection{Formation of Copper Fine Wiring on Polyimide Film}

A film with $\mathrm{GACu} / \mathrm{MACu}$ ratio of 5:5 was formed to obtain a uniform copper film on a polyimide film, and the film was then laser irradiated. In addition, the deposited copper wiring was subjected to electroless copper plating, and thickening of the copper wiring was studied.

The copper-wire formation process is overviewed in Figure 5. The laser was swept over the mixed-copper-complex film formed on the polyimide film. After that, etching with water/ethanol dissolved the copper complex and left copper remaining on the polyimide film. The polyimide film was immersed in an electroless-copper-plating solution, and plated copper was deposited on the film for various immersion times at $60^{\circ} \mathrm{C}$.

The results of observations (DM and SEM images) of the surface condition are shown in Figure 6. It was also found that 1) copper precipitated in the
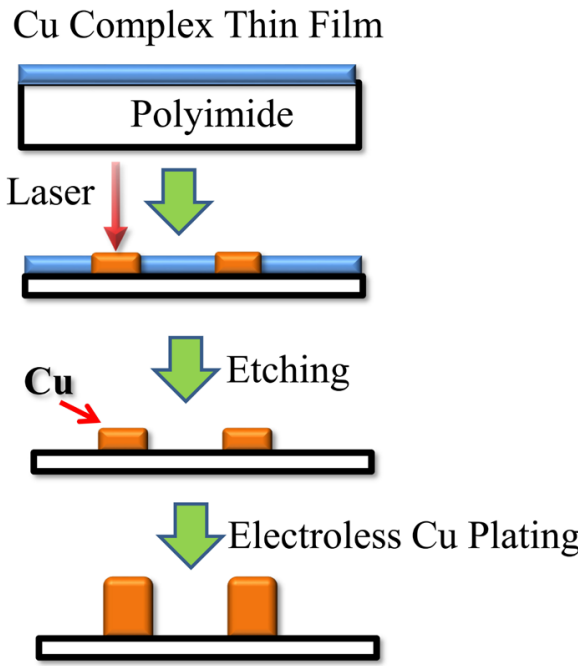

Figure 5. $\mathrm{Cu}$ wire formation process on polyimide film substrate using laser irradiation and electroless $\mathrm{Cu}$ plating. 

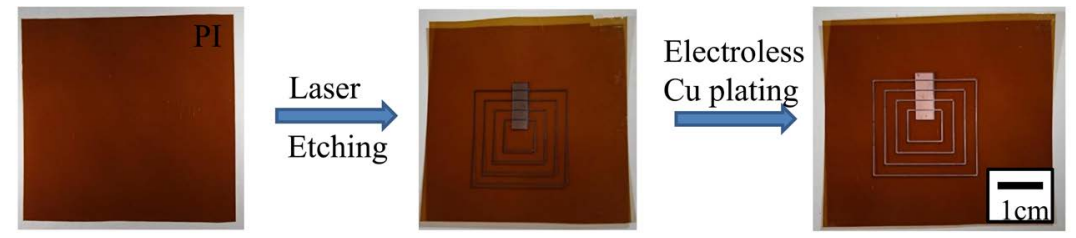

Digital microscope
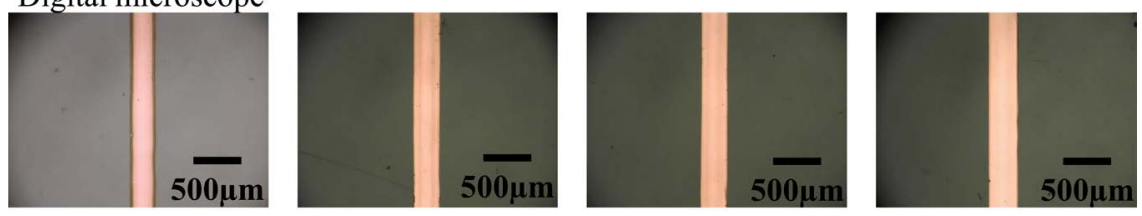

\section{SEM}
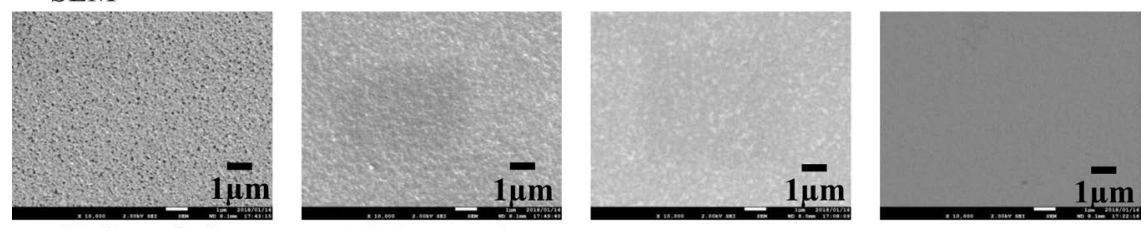

Before plating

Plating 5min.

$10 \mathrm{~min}$.

$20 \mathrm{~min}$.

Figure 6. Surface morphological changes of $\mathrm{Cu}$ wires on polyimide (PI) after electroless $\mathrm{Cu}$ plating.

laser-irradiated part and direct patterning of copper on a polyimide film substrate is thus possible and 2) copper was deposited on this copper wiring by electroless copper plating. It was observed that when the immersion time in the plating solution was increased from 5 to 10 minutes and then from 10 to $20 \mathrm{mi}$ nutes, the copper precipitated more thickly and clearly with time. Looking at the change in the surface condition at that time reveals that the surface of the copper before plating became smoother with immersion time. In the SEM images, minute irregularities are observed in the laser-irradiated film before plating; however, the unevenness decreases with plating time, and it is observed that the surface of the 20-minute-plated film is uniform. This result indicates that the surface becomes smoother as the thickness of the plating film increases. Increasing of plating time enables the densification and smoothness of copper film. Although the width of the wiring on the laser-irradiated film is about $200 \mu \mathrm{m}$, the width tended to increase with plating time, and after 20 minutes, the plating film was about $300 \mu \mathrm{m}$ wide. It is clear that the electroless plating tends to increase not only the thickness of copper film but also the width of the wiring. The adhesion strength of the prepared copper film was high, and the JIS-based-standard tape test (JIS K-5600-5-6) showed excellent adhesion, namely, remaining number/cut number of 100/100.

\subsection{TEM Observation of Cross-Sectional Structure of Copper Wiring}

A TEM photograph of the cross-sectional structure of the copper wiring before plating (fabricated by laser irradiation) is shown in Figure 7 together with the surface-observation image (DM, SEM). A copper thin film is formed on the polyimide film with a thickness of 100 to $200 \mathrm{~nm}$. Although copper particles are not observed in some places, it is presently unknown whether this absence of 


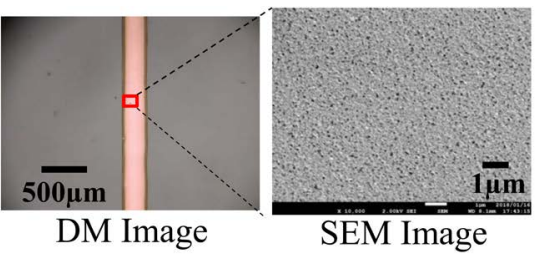

(a) Surface state of $\mathrm{Cu}$ thin film

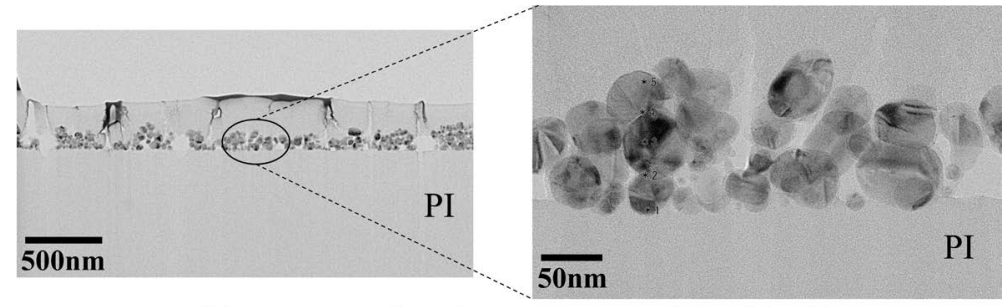

(b) Cross-sectional TEM Image of $\mathrm{Cu}$ thin film

Figure 7. DM image, SEM image and cross-sectional TEM image of $\mathrm{Cu}$ wire prepared on polyimide film substrate using laser irradiation before electroless $\mathrm{Cu}$ plating.

copper particles is due to the characteristic of the laser-irradiated film or missing copper particles at the time the cross-sectional TEM observation sample was prepared. A lot of copper particles with size of 50 to $60 \mathrm{~nm}$ can be seen. And smaller particles, with size of about $10 \mathrm{~nm}$ can also be seen. The precipitated copper particles precipitate in such a manner as to dig into the surface of the polyimide film. This phenomenon is presumed to be because copper precipitated on the parts where the polyimide surface was softened by the heat due to the laser irradiation. It is speculated that the adhesion strength of the deposited copper thin film is high due to the anchor effect of the copper particles biting into the polyimide surface.

The results of elemental analysis by EDX of the deposited copper wiring are shown in Figure 8. A peak corresponding to metal copper is observed at each of observation points (Points 1 to 5), so it is concluded that the precipitated particles are represented by these metal-copper peaks. At Point 1 , close to the polyimide film, elements such as carbon, nitrogen, and oxygen are observed. These elements are presumed to be derived from the constituent molecules of the polyimide film. At Points 3, 4, and 5, nitrogen and oxygen are not observed, but a small amount of carbon is observed; however, this carbon is seemingly derived from the protective film used when the TEM sample was prepared.

A TEM image of the cross-sectional structure of the thin film (plating time: 5 minutes at $60^{\circ} \mathrm{C}$ ), electrolessly plated on copper wiring deposited by laser, is shown in Figure 9. It can be seen that while the plating time is as short as five minutes, a dense plating film was formed on the copper wiring deposited by laser. The film thickness ranged from 200 to $360 \mathrm{~nm}$ depending on the observation point, and the average thickness was about $300 \mathrm{~nm}$. Looking at the form of the precipitated copper particles (see the enlarged view in the TEM image) reveals that the copper particles near the interface with the polyimide film are small, but the copper particles get larger toward the upper layer. The upper layer portion is 

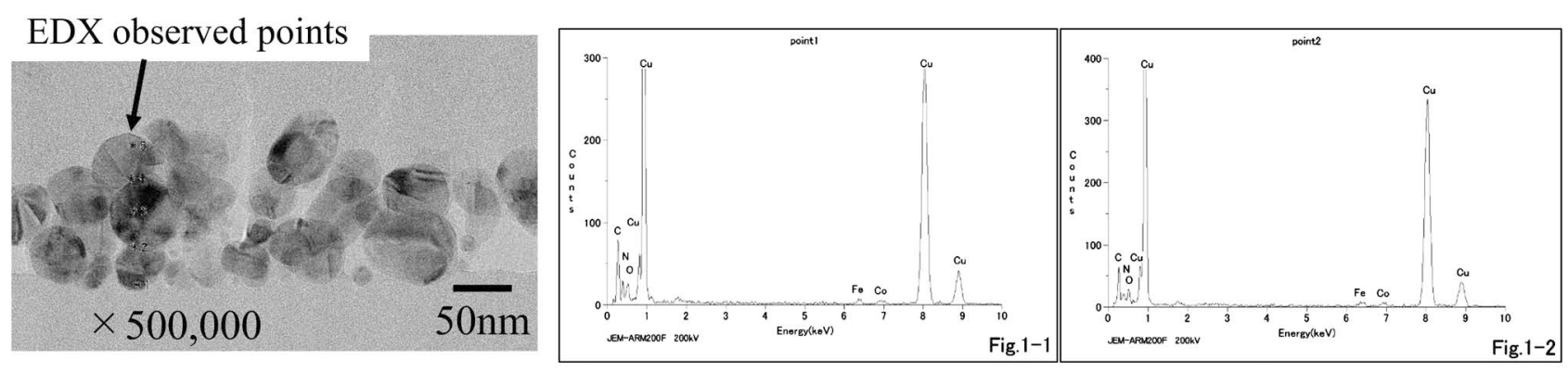

EDX observed area

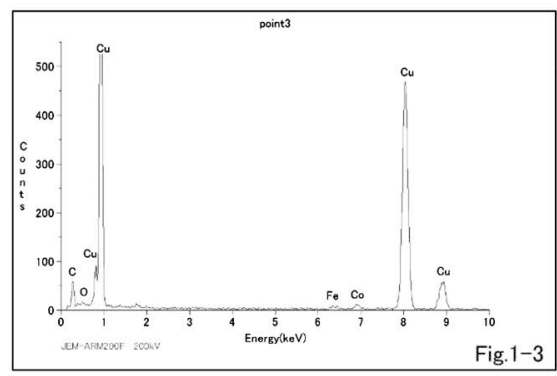

point 3

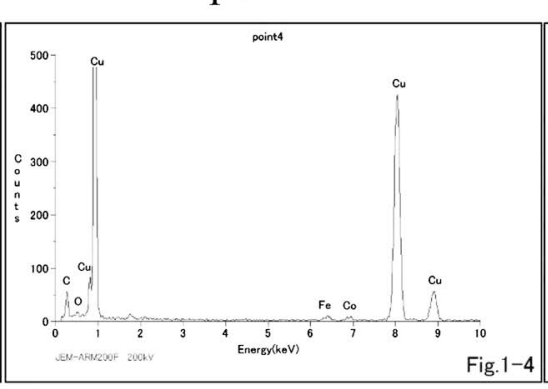

point 4 point 2

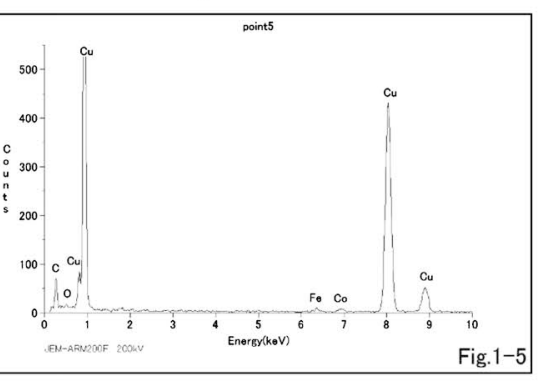

point 5

Figure 8. TEM image and EDX elemental analysis of $\mathrm{Cu}$ wire prepared on polyimide film substrate using laser irradiation.
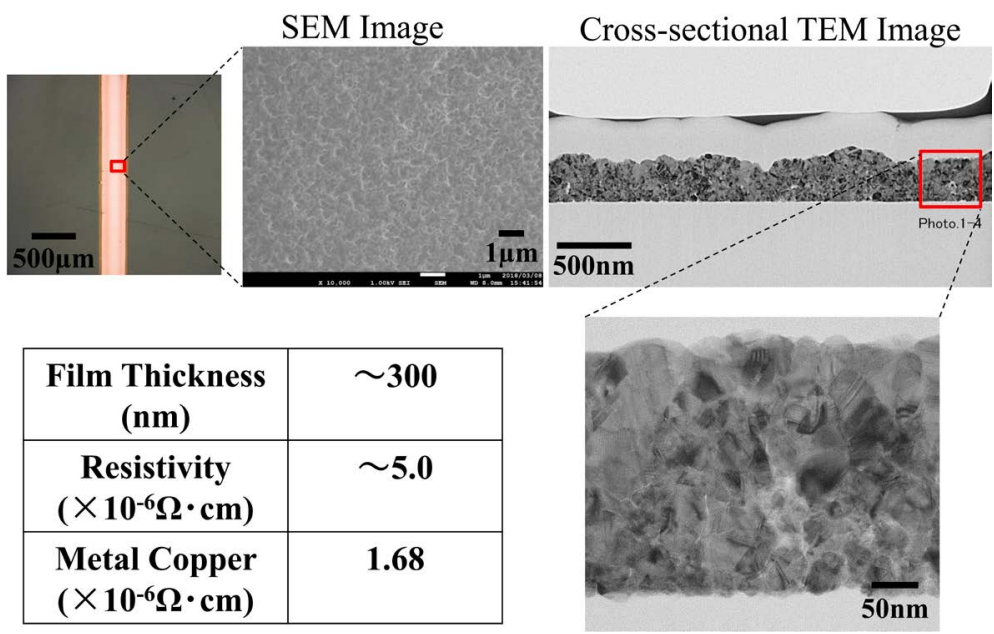

Figure 9. SEM and cross-sectional TEM images of $\mathrm{Cu}$ wire prepared on polyimide film substrate using laser irradiation after electroless Cu plating.

considered to be copper particles precipitated by the electroless copper plating.

From the above results, it is concluded that a plating of copper is formed on the copper formed by laser irradiation. Electroless copper plating usually uses palladium (which is a noble metal) as a catalyst. By applying the method developed in this research, an electroless-copper-plated film can be formed using copper (which is less expensive than palladium) as a catalyst. The resistivity of the plated copper film was about $5 \times 10^{-6} \Omega \cdot \mathrm{cm}$. Although this value is slightly higher than the resistivity of the copper bulk $\left(1.68 \times 10^{-6} \Omega \cdot \mathrm{cm}\right)$, when the plating time was increased to 10 and then 20 minutes, the thickness of the copper film increased and became the same as the resistivity of metallic copper (bulk 
body). As described above, copper wiring can be directly patterned as a film on the PI film in air by laser irradiation to the stable copper complex coating film, and thick copper wiring can be formed on this film by electroless plating; consequently, it is possible to form high-conductivity wiring in air. This result indicates that processing in an inert gas and high-vacuum equipment are unnecessary and that copper wiring can be formed by laser irradiation to a stable copper-complex film on a polyimide film at high-speed. Since the developed method can be applied in air and enables the high-speed deposition of fine copper wiring on a polyimide film by a simple printing process, this method is effective for forming copper wiring on flexible printed circuit board as a low-cost method.

\section{Conclusion}

Formation of copper wiring on a polyimide film by laser irradiation to a stable copper-complex film in air was studied. The results of the study show that metallic copper precipitates on the polyimide film even in air. This copper was generated only in the laser-irradiated parts, so direct patterning of copper wiring was possible. It was also found that copper was precipitated by electroless copper plating on the laser-deposited copper wiring, and it was possible to thicken the copper wiring by this precipitation. The resistivity of the copper wiring was almost the same as that of the bulk of metallic copper. The developed method-combining laser irradiation on a copper-complex-coated film and electroless copper plating-can be applied in air; accordingly, it is believed that this method will contribute to reducing the cost of forming copper wiring on a polyimide film and further development of flexible printed wiring boards.

\section{Conflicts of Interest}

The authors declare no conflicts of interest regarding the publication of this paper.

\section{References}

[1] Perelaer, J., Smith, P.J., Mager, D., Soltman, D., Volkman, S.K., Subramarian, V., Korvink, J.G. and Schubert, U.S. (2010) Printed Electronics. Journal of Materials Chemistry, 20, 8446-8453. https://doi.org/10.1039/c0jm00264j

[2] Yokoyama, M. and Kamata, T. (2008) Advanced Science and Technology for Printable Organic Electronics. CMC Publishers, Tokyo.

[3] Minemawari, H., Yamada, T., Matsui, H., Tsutsumi, J., Haas, S., Chiba, R., Kumai, R. and Hasegawa, T. (2011) Inkjet Printing of Single-Crystal Films. Nature, 475, 364-367. https://doi.org/10.1038/nature10313

[4] Fukuda, K., Sekina, T., Kobayashi, Y., Takeda, Y., Shimizu, M., Yamashita, N., Kumaki, D., Itoh, M., Nagaoka, M., Toda, T., Saito, S., Kurihara, M., Sakamoto, M. and Tokita, S. (2012) Organic Integrated Circuits Using Room-Temperature Sintered Silver Nanoparticles as Printed Electrodes. Organic Electronics, 13, 3296-3301.

[5] Tokuno, T., Nogi, M., Karakawa, M., Jiu, J.T.T., Nge, Y., Aso, K. and Suganuma, K. 
(2011) Fabrication of Silver Nanowire Transparent Electrodes at Room Temperature. Nano Research, 4, 1215-1222. https://doi.org/10.1007/s12274-011-0172-3

[6] Hosel, M. and Krebs, F.C. (2012) Large-Scale Roll-to-Roll Photonic Sintering of Flexo Printed Silver Nanoparticle Electrodes. Journal of Materials Chemistry, 22, 15683-15688.

[7] Nakamoto, M., Kashiwagi, Y. and Yamamoto, M. (2005) Synthesis and Size Regulation of Gold Nanoparticles by Controlled Thermolysis of Ammonium Gold (I) Thiolates in the Absence or Presence of Amines. Inorganica Chimica Acta, 358, 4229-4236. https://doi.org/10.1016/j.ica.2005.03.037

[8] Suganuma, K., Sakamoto, S., Kagami, N., Wakuda, D., Kim, K.S. and Nogi, M. (2012) Low-Temperature Low-Pressure Die Attach with Hybrid Silver Particle Paste. Microelectronics Reliability, 52, 375-380. https://doi.org/10.1016/j.microrel.2011.07.088

[9] Morita, T., Ide, E., Yasuda, Y., Hirose, A. and Kobayashi, K.F. (2008) Study of Bonding Technology Using Silver Nanoprticles. Japanese Journal of Applied Physics, 47, 6615. https://doi.org/10.1143/JJAP.47.6615

[10] Perelaer, J., Abbel. R., Wtinscher, S., Jani, R., van Lammeren, T. and Schubert, U.S. (2012) Roll-to-Roll Compatible Sintering of Inkjet Printed Features by Photonic and Microwave Exposure. Advanced Materials, 24, 2620-2625.

https://doi.org/10.1002/adma.201104417

[11] Kim, Y., Yoo, B.W., Anthony, J.E. and Park, S.K. (2012) Controlled Deposition of a High-Performance Small-Molecule Organic Single-Crystal Transistor Array by Direct Inkjet Printing. Advanced Materials, 24, 497-502.

https://doi.org/10.1002/adma.201103032

[12] Choi, Y., Lee, J.H., Kim, S.J., Yoon, D.H. and Byun, Y.H. (2012) Highly Conductive Polymer-Decorated Cu Electrode Film Printed on Glass Substrates with Novel Precursor-Based Ink and Paste. Journal of Materials Chemistry, 22, 3624-3631. https://doi.org/10.1039/c2jm15124c

[13] Ryu, J.G., Kim, H.S. and Hahn, H.T. (2011) Reactive Sintering of Copper Nanoparticles Using Intense Pulsed Light for Printed Electronics. Journal of Electronic Materials, 40, 42-50. https://doi.org/10.1007/s11664-010-1384-0

[14] Ishizaki, T. and Watanabe, R. (2012) A New One-Pot Method for the Synthesis of $\mathrm{Cu}$ Nanoparticles for Low Temperature Bonding. Journal of Materials Chemistry, 22, 25198-25206. https://doi.org/10.1039/c2jm34954j

[15] Ohishi, T. and Kimura, R. (2015) Fabrication of Copper Wire Using Glyoxylic Acid Copper Complex and Laser Irradiation in Air. Materials Sciences and Applications, 6, 799-808. https://doi.org/10.4236/msa.2015.69082

[16] Ohishi, T. and Takahashi, N. (2018) Fabrication of Fine Copper Wire in Air by Laser Irradiation and Micro-Contact Printing Using Glyoxylic-Acid Copper Complex as Starting Material. Frontiers in Nanoscience and Nanotechnology, 4, 1. https://doi.org/10.15761/FNN.1000165

[17] Shin, D.-H., Woo, S., Yem, H., Cha, M., Cho, S., Kang, M., Jeong, S., Kim, Y., Kang, K. and Piao, Y. (2014) A Self-Reducible and Alcohol-Soluble Copper-Based Metal-Organic Decomposition Ink for Printed Electronics. ACS Applied Materials \& Interfaces, 6, 3312-3319. https://doi.org/10.1021/am4036306

[18] Wang, B.Y., Yoo, T.H., Song, Y.W., Lim, D.S. and Oh, Y.J. (2013) Cu Ion Ink for a Flexible Substrate and Highly Conductive Patterning by Intensive Pulsed Light Sintering. ACS Applied Materials \& Interfaces, 5, 4113-4119.

https://doi.org/10.1021/am303268k 
[19] Araki, T., Sigahara, T., Jiu, J., Nagao, S., Nogi, M., Koga, H., Uchida, H., Shinozaki, K. and Suganuma, K. (2013) Cu Salt Ink Formulation for Printed Electronics Using Photonic Sintering. Langmuir, 29, 11192-11197. https://doi.org/10.1021/la402026r

[20] Ghosh, M.K. and Mittal, L. (1996) Polyimides: Fundamental Aspects and Technological Application. Marcel Dekker, New York.

[21] Numakura, D. (2001) Coom's Printed Circuits Handbook. 5th Edition, McCrow-Hill, New York, NY, 56-60.

[22] Osaka, T., Nakajima, E., Tamiya, Y., Koiwa, I. and Utsuki, K. (1986) Metalization of AlN Ceramics by Electroless Ni-P Plating. Journal of The Electrochemical Society, 133, 2345-2349. https://doi.org/10.1149/1.2108405

[23] Murphy, M.M., Vanherle, J., McEvoy, A.J. and Thampi, K.R. (1994) Electroless Deposition of Electrodes in Solid-Oxide Fuel Cells. Journal of The Electrochemical Society, 141, L94-L96.

[24] Shafeev, G.A. and Hoffmann, P. (1999) Light-Enhanced Electroless Cu Deposition on Laser-Treated Polyimide Surface. Applied Surface Science, 138-139, 455-460. https://doi.org/10.1016/S0169-4332(98)00464-4

[25] Saito, T. and Wakabayashi, S. (2017) The Surface Finishing Technologies on Printed Wiring Boards. Journal of The Surface Finishing Society of Japan, 68, 480-487. https://doi.org/10.4139/sfj.68.480

[26] Iida, H. (2017) Technology and Development Trends of the Thin Copper Foil with Carrier for PCB. Journal of The Surface Finishing Society of Japan, 68, 488-493. https://doi.org/10.4139/sfj.68.488

[27] Buffat, P.H. and Borrel, J.P. (1976) Size Effect on the Melting Temperature of Gold Particles. Physical Review A, 13, 2287. https://doi.org/10.1103/PhysRevA.13.2287

[28] Kim, H.S., Dhade, S.R., Shim, D.E. and Hahn, H.T. (2009) Intense Pulsed Light Sintering of Copper Nanoink for Printed Electronics. Applied Physics A, 97, 791. https://doi.org/10.1007/s00339-009-5360-6 